TRANSACTIONS OF THE

AMERICAN MATHEMATICAL SOCIETY

Volume 329, Number 1, January 1992

\title{
GENERALIZED POTENTIALS AND OBSTACLE SCATTERING
}

\author{
RICHARD L. FORD
}

\begin{abstract}
Potential scattering theory is a very well-developed and understood subject. Scattering for Schrödinger operators represented formally by $-\Delta+V$, where $V$ is a generalized function such as a $\delta$-function, is less understood and requires form perturbation techniques. A general scattering theory for a large class of such singular perturbations of the Laplacian is developed.

The theory has application to obstacle scattering. One considers an alternative mathematical model of an obstacle in $\mathbf{R}^{n}$. Instead of representing the obstacle by deleting the region inhabited by the obstacle from $\mathbf{R}^{n}$, the surface of the obstacle is treated as impenetrable. The impenetrable surface is understood to be the limiting case of a sequence of highly spiked potentials whose support converges to the surface of the obstacle and whose peaks grow without bound. The limiting case is identified as a $\delta$-function acting on the surface of the obstacle. Hamiltonians for the limiting case are constructed and the conditions governing the existence and completeness of the associated wave operators are determined through application of the general theory.
\end{abstract}

\section{INTRODUCTION}

The problem of scattering by obstacles has received much attention by many authors over the past twenty-five years or so. Lax and Phillips [13], T. Ikebe [8], A. Shenk [19], Y. Shizuta [20], and Wilcox [23], have all made significant contributions to the field. In 1986 A. G. Ramm [15] published a book Scattering by Obstacles. All of the above authors have studied solutions to various operator equations (usually the wave or Schrödinger equation) in an exterior domain.

We shall instead view the obstacle as a limiting case of highly spiked potentials concentrated on the surface of the obstacle. A significant advantage of our approach is that we will obtain a perturbed Hamiltonian that acts on the original space, $\mathbf{R}^{n}$, rather than on a second restricted space. This allows the application of single-space scattering theories that may not apply in the two-space setting. To view the obstacle in this way requires a scattering theory applicable to highly singular perturbations of the Laplacian. The purpose of this paper is to develop that theory and to provide examples of its application to this type of obstacle scattering.

Many authors [2, 5, 10, 11, 14] have studied singular potential function perturbations of the Laplacian. Our purposes require a theory that allows

Received by the editors November 14, 1989.

1980 Mathematics Subject Classification (1985 Revision). Primary 47A40; Secondary 35P25, $81 \mathrm{~F} 05$. 
generalized function perturbations such as the delta-function where the Hamiltonian is defined by a quadratic form. In this regard there is a result by Davies [4] that has application. Davies studied the eigenfunction expansion of highly singular perturbations of the Laplacian in $R^{3}$. His perturbation was defined by a form sum in the same fashion as constructed in this work. That is, he defined the sum

$$
(H u, v)+\int u(x) \overline{v(x)} V(d x)
$$

where $H$ is the Laplacian and $V$ is any measure on $R^{3}$ satisfying

$$
\int|x-y|^{-1 \pm \varepsilon}|V|(d x) \leq(1+|y|)^{-1-\varepsilon} .
$$

Davies constructs the associated Hamiltonian and develops an eigenfunction expansion that he uses to prove the completeness of the associated wave operator, which he assumes exists.

Within this paper we shall examine a general setting similar to that of Davies. That is, we shall consider the case where the Laplacian, $H$, is perturbed by the form

$$
(A u, A v)=\int_{\mathbf{R}^{n}} u(x) \overline{v(x)}|V|(d x),
$$

where $V$ is a general signed measure on $\mathbf{R}^{n}$ satisfying certain criteria. An appropriate selfadjoint operator shall be constructed and the associated wave operator shall be shown to exist and be complete. Application of our results to obstacle scattering is accomplished by decomposing $V$ into two parts, one of which is the surface measure on the obstacle. This surface measure corresponds to the $\delta$-function acting on the surface. The other remaining portion of the measure corresponds to the potential that acts on a particle both before and after interaction with the boundary of the obstacle. When our results are compared with those of several of the authors referred to above, we find that with this view of the obstacle, most of the previously mentioned existence and completeness results shall hold and in some cases be generalized. We now state our primary scattering theory result.

Theorem 1.1. Let $H=-\Delta$ and let the signed measure, $V$, on $\mathbf{R}^{n}$ satisfy the following criteria:

$$
\begin{gathered}
\sup _{y} \int_{|x-y| \leq \delta}|x-y|^{2-n}|V|(d x) \rightarrow 0 \text { as } \delta \searrow 0 \\
\int_{|x-y|>1}|x-y|^{(1-n) / 2}|V|(d x) \quad \text { is bounded and } \rightarrow 0 \text { as }|y| \rightarrow \infty
\end{gathered}
$$

and

$$
\int_{|x-y| \leq 1}|x-y|^{2-n}|V|(d x) \quad \text { is bounded and } \rightarrow 0 \text { as }|y| \rightarrow \infty .
$$

As an alternative to (1.4), suppose $V$ satisfies

$$
\iint_{|x-y|>1}|x-y|^{1-n}|V|(d x)|V|(d y)<\infty .
$$

Under the conditions on $V$ above, the following hold: 
(1) There exists a selfadjoint operator, $\widetilde{H}_{1}$, that satisfies

$$
\left(u, \widetilde{H}_{1} v\right) k=(H u, v)+\int u(x) \overline{v(x)} V(d x), \quad u \in D(H), v \in D\left(\widetilde{H}_{1}\right) .
$$

(2) The wave operator, $W\left(\widetilde{H}_{1}, H\right)$, exists and is strongly complete.

We now consider a general bounded obstacle, $\Omega$, in $\mathbf{R}^{n}$ and denote by $V_{1}$ the inherited surface measure. We let $q(x)$ be a real-valued potential and define the measure $V_{2}$ by $V_{2}(S)=\int_{S} q(x) d x$. We require that $V_{2}$ satisfy the conditions of Theorem 1.1. We also require that $\partial \Omega$ be piecewise $C^{2}$. One shows easily (see [6]) that (1.3), (1.4), and (1.5) hold for $V=V_{1}$. We now take $H$ to be the standard selfadjoint realization of the Laplacian in $\mathbf{R}^{n}$. Setting $V=V_{1}+V_{2}$ in Theorem 1.1 we observe that all of the conditions of Theorem 1.1 apply. Thus there exists a selfadjoint operator, $H_{1}$, that corresponds to the formal operator $H+q+\delta$, where $\delta$ is the generalized $\delta$-function on the surface of $\Omega$. More precisely, we have the following

Theorem 1.2. Let $H=-\Delta$, and let $\delta$ be the unweighted $\delta$-function on the surface of an obstacle, $\Omega$, whose boundary, $\partial \Omega$, is piecewise $C^{2}$. Let $q(x)$ be as above. Then there exists a selfadjoint operator, $H_{1}$, that satisfies for all $u \in D(H)$ and $v \in D\left(H_{1}\right)$,

$$
\left(u, H_{1} v\right)=(H u, v)+\int_{\partial \Omega} u(x) \overline{v(x)} d \omega+\int_{\mathbf{R}^{n}} u(x) \overline{v(x)} q(x) d x .
$$

Furthermore,

$$
R_{1}(z)=R(z)+[A R(\bar{z})]^{*} G(z)^{-1} E A R(z),
$$

where $R_{1}(z)$ is the resolvent of $H_{1}, R(z)$ is the resolvent of $H$, and $A, E$, and $G$ are as defined in the proof of Theorem 1.1. In addition, the associated wave operator,

$$
W\left(H_{1}, H\right)=\lim _{t \rightarrow \infty} e^{i H_{1} t} e^{-i H t},
$$

exists and is strongly complete.

This result corresponds with the bounded obstacle results of Weder and Combes [22] and generalizes the results of Ikebe [8]. In applying our result to unbounded obstacles we need to require that the surface measure obeys (1.3). By applying a nonvanishing weighting function, $\rho(x)=(1+|x|)^{-\alpha}$, to the delta function on the surface we can be certain that (1.4) and (1.5) hold for $\alpha>n$. We thus have the following.

Theorem 1.3. Let $\Omega$ be any open subset of $\mathbf{R}^{n}$ with finitely many connected components such that $\partial \Omega$ is a $C^{2}$-manifold of dimension $n-1$ and such that

$$
\sup _{y} \int_{|x-y| \leq \delta}|x-y|^{2-n} d \omega_{x} \rightarrow 0 \text { as } \delta \searrow 0,
$$

where $d \omega$ is the usual inherited surface measure on $\partial \Omega$. For $\alpha>n$, set $\rho(x)=(1+|x|)^{-\alpha}$. Let $V$ be the weighted surface measure given by

$$
V(d x)=\rho(x) d \omega_{x} .
$$


Then there exists a selfadjoint operator, $H_{1}$, on $L^{2}\left(\mathbf{R}^{n}\right)$ satisfying

$$
\left(u, H_{1} v\right)=(H u, v)+\int u(x) \overline{v(x)} V(d x), \quad u \in D(H), v \in D\left(H_{1}\right),
$$

where $H$ is the standard selfadjoint realization of $-\Delta$ in $\mathbf{R}^{n}$. Furthermore, the associated wave operator, $W\left(H_{1}, H\right)$, exists and is strongly complete.

This result extends [3] and [22] to more general unbounded obstacles.

\section{SCATTERING AND COMPACTNESS CRITERIA}

We now provide the statements of the general theorems that are used to prove the concrete theorem of the previous section. We have

Theorem 2.1. Let $A$ be a linear operator from some Hilbert space, $\mathbf{H}$, to another Hilbert space, $\mathbf{K}$. Let $H$ be a selfadjoint operator on $\mathbf{H}$ such that $D(H) \subset$ $D(A)$, and let $E$ be a bounded selfadjoint operator on $\mathbf{K}$. Assume there is a point $z_{0}$ in the resolvent set $\rho(H)$ of $H$ such that the following statements hold for $z=z_{0}$ and $z=\bar{z}_{0}$ :

(a) $A R(z) \in B(\mathbf{H}, \mathbf{K})$, where $R(z)=(z-H)^{-1}$.

(b) $R\left([A R(z)]^{*}\right) \subset D(A)$ and $Q(z)=A(A R(\bar{z}))^{*} \in B(K)$.

(c) $G(z)=1-Q(z)$ and $\widetilde{G}(z)=1-E Q(z)$ have bounded inverses on $\mathbf{K}$.

(d) $Q(z)^{*}=Q(\bar{z})$.

(e) There exists an open set $\Lambda \subset \mathbf{R}$ such that the Lebesgue measure of $(\mathbf{R}-\Lambda)$ is 0 and the following hold:

(1) $Q(z)$ is uniformly continuous in $\omega_{I}$ for each $I \subset \subset \Lambda$, where $A \subset \subset B$ means $A \subset B$ and $\bar{A} \subset B$ and where $\omega_{I}=\left\{(s+i a): s \in I, a<c_{0}\right\}$ for some $c_{0}>0$.

(2) There is a $z_{1} \in \rho(H)$ such that $A R(z)\left[A R\left(\overline{z_{1}}\right)\right]^{*}$ is a compact operator on $\mathbf{K}$ for every $z \in \omega_{I}$.

Then the following conclusions hold:

1. $\widetilde{R}_{1}(z)$ given by

$$
\widetilde{R}_{1}(z)=R(z)+[A R(\bar{z})]^{*} \widetilde{G}(z)^{-1} E A R(z), \quad \widetilde{G}(z)=1-\widetilde{Q}(z),
$$

is the resolvent of a selfadjoint operator, $\widetilde{H}_{1}$, on $\mathbf{H}$ such that $D\left(\widetilde{H}_{1}\right) \subset D(A)$.

2. $\widetilde{H}_{1}$ satisfies

$$
\left(u, \widetilde{H}_{1} v\right)=(H u, v)+(A u, E A v)_{\mathbf{K}}, \quad u \in D(H), v \in D\left(\widetilde{H}_{1}\right) .
$$

3. The wave operator, $W\left(\widetilde{H}_{1}, H\right)$, exists and is strongly complete.

In addition to the preceding abstract theorem we shall prove and use the following compactness theorem.

Theorem 2.2. Let $H$ be the Laplacian on $\mathbf{R}^{n}$ and $V$ be any measure on $\mathbf{R}^{n}$ satisfying

$$
\sup _{y} \int_{|x-y|<1}|x-y|^{2-n}|V|(d x) \quad \text { vanishes as }|y| \rightarrow \infty
$$

and

$$
\sup _{y \in \Gamma} \int_{|x-y|<\delta}|x-y|^{2-n}|V|(d x) \rightarrow 0 \text { as } \delta \searrow 0 \text {, }
$$


where $\Gamma$ is the support of $V$. If $A$ is the inclusion map from $L^{2}\left(\mathbf{R}^{n}\right)$ to $L^{2}\left(\mathbf{R}^{n}:|V|\right)$ with domain $D(H)$, and $R(z)$ is the resolvent for $H$, then for any $z_{1}$ and $z_{2}$ such that im $z_{i} \neq 0$, the operator $A R\left(z_{1}\right)\left[A R\left(\overline{z_{2}}\right)\right]^{*}$ is compact.

We proceed directly to the proofs.

\section{THE PROOFS}

We begin by proving Theorem 2.1. We employ the following theorems due to Schechter [18].

Theorem 3.1. Let $A$ and $B$ be linear operators from some Hilbert space, $\mathbf{H}$, to another Hilbert space, $\mathbf{K}$. Let $H$ be a selfadjoint operator on $\mathbf{H}$ such that $D(H) \subset D(A) \cap D(B)$. Assume there is a point $z_{0}$ in the resolvent set $\rho(H)$ of $H$ such that the following statements hold for $z=z_{0}$ and $z=\overline{z_{0}}$ :

(a) $A R(z), B R(z) \in B(\mathbf{H}, \mathbf{K})$, where $R(z)=(z-H)^{-1}$.

(b) $R\left([A R(z)]^{*}\right) \subset D(B)$ and $Q(z)=B[A R(\bar{z})]^{*} \in B(K)$.

(c) $G(z)=1-Q(z)$ has a bounded inverse on $\mathbf{K}$.

(d) $T(z)^{*}=T(\bar{z})$ for $T(z)=R(z)+[A R(\bar{z})]^{*} G(z)^{-1} B R(z)$.

Then $T(z)$ is the resolvent of a selfadjoint operator, $H_{1}$, on $\mathbf{H}$ such that $D\left(H_{1}\right) \subset D(B)$. Furthermore, $H_{1}$ satisfies

$$
\left(u, H_{1} v\right)=(H u, v)+(A u, B v)_{\mathbf{K}} \quad \forall u \in D(A), v \in D(B) .
$$

Theorem 3.2. Suppose that

(a) (3.1) holds and and

(b) there is an open set, $\Lambda \subset \mathbf{R}$, such that $(\mathbf{R}-\Lambda)$ has Lebesgue measure 0

(1) (a)-(d) of Theorem 3.1 holds for all $z \in \omega_{\Lambda}$.

(2) $\lim \sup _{a \searrow 0} \sup _{s \in I} a\|A R(s+i a)\|^{2}+a\|B R(s+i a)\|^{2}<\infty$ for each $I \subset \subset$ $\Lambda$.

(3) $Q(z)$ is uniformly continuous in $\omega_{I}$ for each $I \subset \subset \Lambda$.

(4) There is a $z_{1} \in \rho(H)$ such that $B R(z)\left[A R\left(\overline{z_{1}}\right)\right]^{*}$ is a compact operator on $\mathbf{K}$ for every $z \in \omega_{\Lambda}$.

Then the wave operator, $W\left(H_{1}, H\right)$, exists and is strongly complete.

To prove our Theorem 2.1 we first provide the following technical lemmas.

Lemma 3.3. Let conditions (a), (b), and (d) of Theorem 2.1 hold for $z=z_{0}$ and $z=\bar{z}_{0}$. Then those conditions also hold for all $z \in \rho(H)$.

Proof. For arbitrary $z \in \rho\left(H_{1}\right)$ we have, by the resolvent equation,

$$
A R(z)=\left(z_{0}-z\right)\left[A R\left(z_{0}\right)\right] R(z)+A R\left(z_{0}\right) .
$$

Thus, $A R(z)$ is bounded for all $z \in \rho(H)$ proving (a) holds for all $z \in \rho(H)$. From (3.2) we also have

$$
[A R(\bar{z})]^{*}=\left(z_{0}-z\right) R(z)\left[A R\left(\bar{z}_{0}\right)\right]^{*}+\left[A R\left(\bar{z}_{0}\right)\right]^{*},
$$

which shows that (b) holds for all $z \in \rho(H)$. As above, for arbitrary $z \in \rho(H)$ to verify that $Q(\bar{z})=Q(z)^{*}$ we have from $(3.3)$

$$
Q(\bar{z})=\left\{\left(z_{0}-z\right) A R\left(z_{0}\right)[A R(\bar{z})]^{*}\right\}^{*}+Q\left(\overline{z_{0}}\right)=Q(z)^{*} .
$$

Thus (d) holds for all $z \in \rho(H)$, completing the lemma. 
Lemma 3.4. Under the conditions of Theorem 2.1,

$$
\widetilde{G}(z)^{-1} E=E\left[\widetilde{G}(\bar{z})^{-1}\right]^{*}, \quad z=z_{0}, \overline{z_{0}} .
$$

To see this, we have, by assumption, $Q(z)^{*}=Q(\bar{z})$ and $\widetilde{G}(z)$ has a bounded inverse for $z=z_{0}, \overline{z_{0}}$, thus we have

and therefore,

$$
E \widetilde{G}(z)^{*}=E[1-Q(\bar{z})] E=\widetilde{G}(\bar{z}) E
$$

$$
E=E \widetilde{G}(\bar{z})^{*}\left[\widetilde{G}(\bar{z})^{-1}\right]^{*}=\widetilde{G}(z) E\left[\widetilde{G}(\bar{z})^{*}\right]^{-1} .
$$

Applying $\widetilde{G}(z)^{-1}$ to both sides we obtain (3.5).

Lemma 3.5. Let the conditions of Theorem 3.1 hold and assume that $B=E A$, where $E$ is a bounded, selfadjoint operator. Then $\widetilde{G}(z)=1-B[A R(\bar{z})]^{*}$ has a bounded inverse for all $z \in \rho(H)$.

Letting $\widetilde{R}_{1}(z)=T(z)$ we remark that by setting $u=R(z) f$ and $v=\widetilde{R}_{1}(z) g$ in (2.2) we have (see [17])

$$
\widetilde{R}_{1}(z)-R(z)=\left[A \widetilde{R}_{1}(\bar{z})\right]^{*} B R(z)=[A R(\bar{z})]^{*} B \widetilde{R}_{1}(z) .
$$

Using this fact at the appropriate moment below for $\widetilde{Q}_{1}(z)=B\left[A \widetilde{R}_{1}(z)\right]^{*}$, we have

$$
\begin{aligned}
{[1-\widetilde{Q}(z)]\left[1+\widetilde{Q}_{1}(z)\right] } & =1-\widetilde{Q}(z)+\widetilde{Q}_{1}(z)-\widetilde{Q}(z) \widetilde{Q}_{1}(z) \\
& =1+E A\left\{A\left[A \widetilde{R}_{1}(z)\right]^{*}[B R(\bar{z})]\right\}^{*}-\widetilde{Q}(z) \widetilde{Q}_{1}(z) \\
& =1+B[A R(\bar{z})]^{*} E A\left[A \widetilde{R}_{1}(\bar{z})\right]^{*}-\widetilde{Q}(z) \widetilde{Q}_{1}(z) \\
& =1,
\end{aligned}
$$

where we have used the fact that

$$
\left\{A\left[A \widetilde{R}_{1}(z)\right]^{*}\right\}^{*}=\left\{A\left[A \widetilde{R}_{1}(\bar{z})\right]^{*}\right\} .
$$

This fact follows from a long but straightforward calculation when $z=z_{0}$ and $z=\bar{z}_{0}$ in (2.1), from the conditions on $E$, and from condition (c) of Theorem 3.1. For arbitrary $z \in \omega_{I}$ we follow the same calculation as in (3.4).

Lemma 3.6. Let $R(z)$ be the resolvent of a selfadjoint operator and suppose $A R(z)$ is bounded and $R\left([A R(z)]^{*}\right) \subset D(A)$ for $\operatorname{im} z \neq 0$. Then for $z=s+i a$, $a>0$,

$$
A R(z)[A R(z)]^{*}=\frac{-1}{2 i a}\left\{A[A R(\bar{z})]^{*}-A[A R(z)]^{*}\right\} .
$$

Proof. This equality follows easily from the first resolvent equation applied to the R.H.S. of (3.10). We have the following corollary.

Corollary 3.7. If $\sup _{z \in \omega_{I}}\left\|A[A R(\bar{z})]^{*}\right\|=c<\infty$ and $Q(z)^{*}=Q(\bar{z})$, then

$$
\sup _{z \in \omega_{I}} a\|A R(s+i a)\|^{2} \leq c<\infty \text {. }
$$

Proof. We have

$$
\begin{aligned}
a\left\|[A R(s+i a)]^{*} f\right\|^{2} & =a\left(A R(s+i a)[A R(s+i a)]^{*} f, f\right) \\
& \leq \frac{1}{2}\left\{\left\|A[A R(s-i a)]^{*}\right\|+\left\|A[A R(s+i a)]^{*}\right\|\right\}\|f\|^{2} \\
& \leq\|Q(z)\|\|f\|^{2} .
\end{aligned}
$$

The corollary follows from this estimate 
Proof of Theorem 2.1. Note all of the conditions in Theorem 3.1 are assumed in the hypothesis of Theorem 2.1 with the exception of $(d)$. To verify that (d) holds as well, we note that by Lemma 3.4

$$
\begin{aligned}
T(z)^{*} & =\left\{R(z)+[A R(\bar{z})]^{*} \widetilde{G}(z)^{-1} E A R(z)\right\}^{*} \\
& =R(\bar{z})+[A R(z)]^{*} \widetilde{G}(\bar{z})^{-1} E A R(\bar{z})=T(\bar{z}) .
\end{aligned}
$$

This completes the verification that all of the conditions of Theorem 3.1 hold. We thus can conclude that the appropriate Hamiltonian exists satisfying (2.1) and (2.2), so that (a) of Theorem 3.2 is satisfied. In addition, we can now apply Lemmas 3.3 and 3.5. This together with the fact that $E$ is bounded allows us to conclude that $(\mathrm{b})(1)$ of Theorem 3.2 holds. Since $Q(z)$ is uniformly continuous in $\omega_{I}$, so is $E Q(z)$ and so $(\mathbf{b})(3)$ holds. Furthermore, by Lemma 3.3 the conditions of Lemma 3.6 hold so that we can apply Corollary 3.7, which together with the boundedness of $E$ allows us to conclude that (b)(2) holds. As our hypothesis assumes $A R(z)\left[A R\left(\bar{z}_{1}\right)\right]^{*}$ is compact, therefore so is $E A R(z)\left[A R\left(\bar{z}_{1}\right)\right]^{*}$ since $E$ is bounded. Thus (b)(4) holds, completing the verification that all of the conditions of Theorem 3.2 hold. This completes the proof of Theorem 2.1.

Proof of Theorem 2.2. The proof of this theorem will be accomplished through a series of lemmas. The basic concept is to analyze the kernel associated with $A R\left(z_{1}\right)\left[A R\left(z_{2}\right)\right]^{*}$ and show that the operator associated with a properly truncated Hilbert-Schmidt version of that kernel converges as an operator in the operator norm as the truncations move to infinity. We let $G_{\kappa}(x)$ be the usual Green's function associated with $R(z)$ where $\operatorname{im} \kappa>0$ and $\kappa^{2}=z$ so that $R(z) u(x)=G_{\kappa} * u(x)$. We then have that

$$
\begin{aligned}
& \left(A R\left(z_{1}\right)\left[A R\left(\overline{z_{2}}\right)\right]^{*} u, v\right) \\
& \quad=\iiint G_{\kappa_{1}}(x-\xi) G_{\kappa_{2}}(\xi-y) u(y) \overline{v(x)} V(d y) d \xi V(d x) \\
& \quad=\iint K(x, y) u(y) \overline{v(x)} V(d y) V(d x),
\end{aligned}
$$

where

$$
K(x, y)=\int_{\mathbf{R}^{n}} G_{\kappa_{1}}(x-\xi) G_{\kappa_{2}}(\xi-y) d \xi
$$

From Thoe [21] we have the following estimates:

$$
\left|G_{\kappa}(x)\right| \leq \begin{cases}c_{R}|\kappa|^{(n-3) / 2}|x|^{(1-n) / 2} e^{-|x| \text { im } \kappa} & \text { if }|x| \geq R, \\ c_{R}|x|^{2-n} & \text { if }|x|<R,\end{cases}
$$

where $c_{R}$ is a constant depending only on $R$. From this it is easily verified that $K(x, y)$ satisfies the following estimates:

$$
|K(x, y)| \leq \begin{cases}K_{R}|x-y|^{2-n} & \text { if }|x-y|<R \\ K_{R}|x-y|^{(1-n) / 2} e^{-|x-y| \kappa} & \text { otherwise }\end{cases}
$$

where $\kappa=\min \left(\operatorname{im} \kappa_{1}, \operatorname{im} \kappa_{2}\right)$. We let $C=\sup _{y} \int_{|x-y|<1}|x-y|^{2-n}|V|(d x)$. Then by standard arguments one can show there is some constant, $M$, such that for any $R>1$,

$$
\int_{|x-y|<R}|x-y|^{2-n}|V|(d x)<C M R^{n}
$$


Furthermore, $M$ can be chosen so that

$$
\int_{|x-y|<R}|V|(d x)<C M R^{n}
$$

holds as well. We now set $B_{N}^{y}=\{x:|x-y|<N\}$ and let $T_{N}^{y}=B_{N+1}^{y}-B_{N}^{y}$. We have

Lemma 3.8. There are constants, $k_{1}$ and $k_{2}$, such that for any $N>0$,

$$
\int_{T_{N}^{y}}|K(x, y)||V|(d x) \leq k_{1} e^{-k_{2} N}(N+1)^{n} .
$$

Proof. From (3.16) we have that $|K(x, y)| \leq K_{2} e^{-k_{2}|x-y|}$ for $|x-y| \geq 2$, where $k_{2}=\min \left(\operatorname{im} \kappa_{1}, \operatorname{im} \kappa_{2}\right)$. Thus using (3.14) we have that

$$
\begin{aligned}
\int_{T_{N}^{y}}|K(x, y)||V|(d x) & \leq \sup _{x \in T_{N}^{y}}|K(x, y)| \int_{T_{N}^{y}}|V|(d x) \\
& \leq K_{2} e^{-k_{2} N} \int_{B_{N+1}^{y}}|V|(d x) \\
& \leq C M(N+1)^{n} K_{2} e^{-k_{2} N} .
\end{aligned}
$$

Setting $k_{1}=C M K_{2}$ completes our lemma.

Lemma 3.9. For each fixed $N, S_{R}^{N}$ vanishes as $R \rightarrow \infty$, where

$$
S_{R}^{N}=\sup _{|y|>R} \int_{B_{N}^{y}}|V|(d x) .
$$

Proof. Set

$$
D_{R}=\sup _{|y|>R} \int_{|x-y|<1}|V|(d x) .
$$

By (2.3) we have that $D_{R}$ vanishes as $R \rightarrow \infty$. Therefore, for $R>N$, since

$$
\sup _{y^{\prime} \in B_{N}^{y}} \int_{\left|x-y^{\prime}\right|<1}|V|(d x) \leq D_{R-N}
$$

and using the fact that $B_{N}^{y}$ can be covered by $M N^{n}$ unit balls whose centers are in $B_{N}^{y}$, we have that

$$
S_{R}^{N} \leq M N^{n} \sup _{|y|>R-N} \int_{|x-y|<1}|V|(d x)=D_{R-N} M N^{n} .
$$

The R.H.S. vanishes as $R \rightarrow \infty$.

Lemma 3.10. Let $N$ be fixed. Then $T_{R} \rightarrow 0$ as $R \rightarrow \infty$, where

$$
T_{R}=\sup _{|y|>R} \int_{B_{N}^{y}}|K(x, y)||V|(d x) .
$$

Proof. We write $T_{R}=I_{R}+J_{R}$, where

$$
I_{R}=\sup _{|y|>R} \int_{B_{N}^{y}-B_{1}^{y}}|K(x, y)||V|(d x)
$$


and

$$
J_{R}=\sup _{|y|>R} \int_{B_{1}^{y}}|K(x, y)||V|(d x) .
$$

$J_{R}$ vanishes as $R \rightarrow \infty$ by our assumption (2.3) and our estimates (3.16) on $K$. Using the estimates on $K$ we also have

$$
\begin{aligned}
I_{R} & \leq \sup _{|y|>R} \int_{B_{N}^{y}-B_{1}^{y}} K_{1}|x-y|^{(1-n) / 2} e^{-|x-y| \kappa}|V|(d x) \\
& \leq \sup _{|y|>R} \int_{B_{N}^{y}} K_{1}|V|(d x)=K_{1} S_{R}^{N}
\end{aligned}
$$

which also vanishes as $R \rightarrow \infty$ by Lemma 3.9 .

Lemma 3.11. If the measure $V$ on $\mathbf{R}^{n}$ satisfies (2.3), then $K(x, y)$ satisfies

$$
\sup _{|y|>R} \int|K(x, y)||V|(d x) \rightarrow 0 \text { as } R \rightarrow \infty \text {. }
$$

Proof. We let $\varepsilon>0$ be given and choose $M$ so large that

$$
\sum_{N=M}^{\infty} k_{1} e^{-k_{2} N}(N+1)^{n}<\frac{\varepsilon}{2}, \quad M \geq 2 .
$$

We then apply Lemma 3.10 and choose $R$ so large that

$$
|y|>R \Rightarrow \int_{B_{M}^{y}}|K(x, y)||V|(d x)<\frac{\varepsilon}{2} .
$$

Then for any such $y$ we have

$$
\begin{aligned}
\int_{\mathbf{R}^{n}}|K(x, y)||V|(d x) & \leq \int_{B_{M}^{y}}|K(x, y)||V|(d x)+\sum_{N=M}^{\infty} \int_{T_{N}^{y}}|K(x, y)||V|(d x) \\
& \leq \frac{\varepsilon}{2}+\sum_{N=M}^{\infty} k_{1} e^{-k_{2} N}(N+1)^{n} \leq \varepsilon,
\end{aligned}
$$

where the estimations above make use of Lemma 3.8. Our lemma follows.

Lemma 3.12. $\int_{|x-y|>2}|K(x, y)|^{2}|V|(d x)$ is uniformly bounded in $y$.

Proof. Following the proof of Lemma 3.8 we have that

$$
\begin{aligned}
\int_{T_{N}^{y}}|K(x, y)|^{2}|V|(d x) & \leq \sup _{x \in T_{N}^{y}}|K(x, y)|^{2} \int_{T_{N}^{y}}|V|(d x) \\
& \leq k_{1}^{2} e^{-2 k_{2} N}(N+1)^{n} S_{0}^{N}
\end{aligned}
$$

where $k_{1}$ is as in Lemma 3.8 and where $S_{0}^{N}$ is as in (3.21). Since $S_{0}^{N}<\infty$ by Lemma 3.9, we can conclude that

$$
\int_{|x-y|>2}|K(x, y)|^{2}|V|(d x) \leq \sum_{N=3}^{\infty} k_{1}^{2} e^{-2 k_{2} N}(N+1)^{n} S_{0}^{N}<\infty .
$$


We now complete the proof of Theorem 2.2. We set $L=A R\left(z_{1}\right)\left[A R\left(z_{2}\right)\right]^{*}$ and let $L^{n}$ and $L_{m}^{n}$ be the operators with kernels $K^{n}$ and $K_{m}^{n}$ given by

$$
\begin{aligned}
& K^{n}= \begin{cases}K(x, y), & |x-y|>1 / n, \\
0, & \text { otherwise, }\end{cases} \\
& K_{m}^{n}= \begin{cases}K^{n}(x, y), & |y|<m, \\
0, & \text { otherwise } .\end{cases}
\end{aligned}
$$

Our proof consists of showing that $L_{m}^{n}$ is Hilbert-Schmidt and that $L_{m}^{n} \rightarrow L^{n}$ in the operator norm. Having shown that $L^{n}$ is compact, we show $L^{n} \rightarrow L$ in the operator norm. This will complete our proof. To see that $L_{m}^{n}$ is HilbertSchmidt we apply Lemma 3.12 and the fact that $K(x, y)$ is bounded uniformly in $y$ on the set $\{(x, y):|x-y| \in(1 / n, 2]\}$ to conclude

$$
\sup _{y} \int_{|x-y|>1 / n}|K(x, y)|^{2}|V|(d x)<\infty .
$$

Thus,

$$
\begin{aligned}
\iint\left|K_{m}^{n}(x, y)\right|^{2}|V|(d x)|V|(d y) \\
\quad=\int_{|y|<m} \int_{|x-y|>1 / n}|K(x, y)|^{2}|V|(d x)|V|(d y)<\infty .
\end{aligned}
$$

Next, to see $L_{m}^{n} \rightarrow L^{n}$ we have

$$
\begin{aligned}
\left|\left(\left[L_{m}^{n}-L^{n}\right] u, v\right)\right|^{2} & =\left|\iint_{|y|>m} K^{n}(x, y) u(y) \overline{v(x)}\right| V|(d x)| V|(d y)|^{2} . \\
& \leq\|u\|^{2}\|v\|^{2} \sup _{|y|>m}\left\{\int\left|K^{n}(x, y)\right||V|(d y)\right\}^{2} .
\end{aligned}
$$

The integral in (3.33) vanishes as $m \rightarrow \infty$ by Lemma 3.11. Thus, $\left\|L_{m}^{n}-L^{n}\right\| \rightarrow$ 0 as $m \rightarrow \infty$ showing each $L^{n}$ is compact. Similarly,

$$
\left|\left(\left[L-L^{n}\right] u, v\right)\right|^{2} \leq\|u\|^{2}\|v\|^{2} \sup _{x}\left\{\int_{|x-y|<1 / n}|K(x, y)||V|(d y)\right\}^{2} .
$$

We see by our condition (2.4) and the estimate (3.16) that the last integral in (3.34) vanishes as $n \rightarrow \infty$. Thus, $\left\|L-L^{n}\right\| \rightarrow 0$ as $n \rightarrow \infty$ showing $L$ is compact.

\section{Applications}

We now consider applications of the theorems in $\S 2$ to prove the theorem in the first section. Our proof consists of verifying conditions (a)-(e) of Theorem 2.1. We let $\mathbf{H}=L^{2}\left(\mathbf{R}^{n}\right)$ and $\mathbf{K}=L^{2}\left(\mathbf{R}^{n}:|V|\right) . R(z)$ is the resolvent of the Laplacian and $A$ is the inclusion operator from $\mathbf{H}$ to $\mathbf{K}$. The first condition that we verify is that $A R(z)$ is bounded for all $z: \operatorname{im}(z) \neq 0$. Note that this will imply $D(H) \subset D(A)$. 
Lemma 4.1. Let $A$ be as above. Then $A R(z)$ is a bounded operator from $\mathbf{H}$ to $\mathbf{K}$ for all $z$ such that $\operatorname{im}(z) \neq 0$.

Proof. We have by the proof of Lemma 3.11 that

$$
\sup _{y} \int|G(x-y)||V|(d x)=k<\infty
$$

for some constant, $k$. Thus we have the following:

$$
\begin{aligned}
|(A R(z) u, v)|^{2} & \leq\left\{\iint\left|G_{\kappa}(x-y) u(y) v(x)\right||V|(d x)\right\}^{2} \\
& \leq k\|G\|_{L^{1}}\|u\|^{2}\|v\|^{2}
\end{aligned}
$$

This shows that (a) of Theorem 2.1 holds. To show that (b)-(d) hold we provide the following technical lemmas.

Lemma 4.2. For any $r>0$ and re $z<0$ there is a constant, $\alpha$, depending only on $r$ and the dimension, $n$, such that

$$
\left|G_{\kappa}(x)\right| \leq \alpha \mid \text { re }\left.z\right|^{-1 / 2}|x|^{2-n} \text { for all }|x|>r,
$$

where $\kappa^{2}=z$ and $\operatorname{im} \kappa>0$.

Proof. By Schechter [16] we have

$$
G_{\kappa}(x)=\frac{\pi^{-n / 2}}{4}|x|^{2-n} \int_{0}^{\infty} e^{z|x|^{2} / 4 s-s} s^{n / 2-2} d s, \quad \text { re } z<0 .
$$

Thus, for $|x|>r$ we have

$$
\left|G_{\kappa}(x)\right| \leq \frac{\pi^{-n / 2}}{4}|x|^{2-n} \int_{0}^{\infty} e^{\text {re } z r^{2} / 4 s-s} s^{n / 2-2} d s \leq \alpha|x|^{2-n} \mid \text { re }\left.z\right|^{-1 / 2}
$$

where

$$
\alpha=\frac{\pi^{-n / 2}}{4 r} 2^{(2-n) / 2}\{\Gamma(n-1)\}^{1 / 2} .
$$

This completes the lemma.

We now provide the necessary results ensuring the existence of a $z_{0}$ in $\rho(H)$ such that $1-Q(z)$ has a bounded inverse for $z=z_{0}$, and $z=\overline{z_{0}}$ under the hypothesis that $V$ satisfies (1.3) and either (1.4) and (1.5) or (1.5) and (1.6). Under the former hypothesis we have

Lemma 4.3. If $V$ satisfies (1.3), (1.4), and (1.5), then given $z$ such that $\mathrm{re} z<0$ and given any $\varepsilon>0$ there exist constants $\alpha_{1}$ and $\alpha_{2}$ depending only on $\varepsilon$ such that

$$
\sup _{y} \int\left|G_{\kappa}(x-y)\right||V|(d x) \leq \varepsilon+\alpha_{1}|\operatorname{re} z|^{-1 / 2}+\alpha_{2}|\kappa|^{(3-n) / 2} e^{-\operatorname{im} \kappa} .
$$

Proof. Let $\varepsilon>0$ be given. Then by (1.3) and (3.15) there is some $r \in(0,1)$ such that

$$
\sup _{y} \int_{|x-y| \leq r}\left|G_{\kappa}(x-y)\right||V|(d x)<\varepsilon
$$


By Lemma 4.2 we have

$$
\begin{aligned}
& \sup _{y} \int_{r<|x-y|<1}\left|G_{\kappa}(x-y)\right||V|(d x) \\
& \quad \leq \sup _{y} \alpha \mid \text { re }\left.z\right|^{-1 / 2} \int_{|x-y|<1}|x-y|^{2-n}|V|(d x) \leq \alpha_{1} \mid \text { re }\left.z\right|^{-1 / 2},
\end{aligned}
$$

where $\alpha_{1}=\alpha \sup _{y} \int_{|x-y|<1}|x-y|^{2-n}|V|(d x)$, which is finite by (1.5) and independent of $z$. We also have in view of (3.15) and (1.4) that

$$
\sup _{y} \int_{|x-y|>1}\left|G_{\kappa}(x-y)\right||V|(d x) \leq \alpha_{2}|\kappa|^{(3-n) / 2} e^{-\operatorname{im} \kappa},
$$

where

$$
\alpha_{2}=c_{1} \sup _{y} \int_{|x-y|>1}|x-y|^{(1-n) / 2}|V|(d x),
$$

which is finite by (1.4). Combining (4.8), (4.9), and (4.10) completes our lemma.

We can now provide a useful estimate for $\|Q(z)\|$. We write

$$
\begin{aligned}
|(Q(z) u, v)| & \leq\|u\|\|v\|\left\{\sup _{y \in \Gamma} \int\left|G_{\kappa}(x-y)\right| V(d x)\right\} \\
& \leq\|u\|\|v\|\left\{\varepsilon+\alpha_{1} \mid \text { re }\left.z\right|^{-1 / 2}+\alpha_{2}|\kappa|^{(3-n) / 2} e^{-\operatorname{im} \kappa}\right\} .
\end{aligned}
$$

The above yields the estimate

$$
\|Q(z)\| \leq \varepsilon+\alpha_{1} \mid \text { re }\left.z\right|^{-1 / 2}+\alpha_{2}|\kappa|^{(3-n) / 2} e^{-\mathrm{im} \kappa} .
$$

We now consider the case when (1.5) and (1.6) hold and provide estimates for $\|Q(z)\|$. We have

$$
\begin{aligned}
|(Q(z), u, v)|= & \left|\iint G_{\kappa}(x-y) u(y) \overline{v(x)} V(d y) V(d x)\right| \\
\leq & \iint_{|x-y| \leq 1}\left|G_{\kappa}(x-y) u(y) v(x)\right||V|(d y)|V|(d x) \\
& +\iint_{|x-y|>1}\left|G_{\kappa}(x-y) u(y) v(x)\right||V|(d y)|V|(d x) .
\end{aligned}
$$

We let (4.13) and (4.14) be denoted by $I_{1}$ and $I_{2}$ respectively. Regarding $I_{1}$ we can utilize (4.8) and (4.9) and conclude that given any $\varepsilon>0$, there is some $\alpha_{1}$ such that

$$
\sup _{y} \int_{|x-y| \leq 1}\left|G_{\kappa}(x-y)\right||V|(d y)<\varepsilon+\alpha_{1} \mid \text { re }\left.z\right|^{-1 / 2} .
$$

Thus, applying Schwartz to $I_{1}$ we obtain

$$
\begin{aligned}
I_{1}^{2} & \leq\|u\|^{2}\|v\|^{2}\left\{\sup _{y} \int_{|x-y| \leq 1}\left|G_{\kappa}(x-y)\right||V|(d y)\right\}^{2} \\
& =\|u\|^{2}\|v\|^{2}\left\{\varepsilon+\alpha_{1}|\operatorname{re} z|^{-1 / 2}\right\}^{2} .
\end{aligned}
$$

We now apply (3.15) to $I_{2}$ obtaining

$$
I_{2} \leq \alpha_{2}|\kappa|^{(3-n) / 2} e^{-\operatorname{im} \kappa}\|u\|\|v\|,
$$


where

$$
\alpha_{2}=c_{1}\left\{\iint_{|x-y|>1}|(x-y)|^{1-n}|V|(d y)|V|(d x)\right\}^{1 / 2},
$$

which is finite by (1.6).

Combining (4.16) and (4.17) we obtain that

$$
\|Q(z)\| \leq \varepsilon+\alpha_{1}|\operatorname{re} z|^{-1 / 2}+\alpha_{2}|\kappa|^{(3-n) / 2} e^{-\operatorname{im} \kappa},
$$

where $\alpha_{1}$ and $\alpha_{2}$ are independent of $z$. The above shows that (b) of Theorem 2.1 holds. Furthermore, we now provide

Lemma 4.4. If $V$ satisfies (1.3) and either (1.5) and (1.6) or (1.4) and (1.6), then there is a $z_{0} \in \rho(H)$ such that $Q(z)$ has norm less than 1 for $z=z_{0}$ and $z=\overline{z_{0}}$, hence $1-Q(z)$ has a bounded inverse for $z=z_{0}$ and $z=\overline{z_{0}}$.

Proof. By estimates (4.12) and (4.18) we have only to choose $\varepsilon=\frac{1}{2}$ and then choose $z_{0}$ such that $\operatorname{re} z$ and $\operatorname{im} \kappa$ are sufficiently large.

By the above Lemma 4.4 we can find a $z_{0}$ such that condition (c) of Theorem 2.1 is satisfied. To verify condition (d) we have

$$
\begin{aligned}
(Q(\bar{z}) u, v)_{\mathbf{K}} & =\iint G_{\kappa^{\prime}}(x-y) u(y) \overline{v(x)} V(d y) V(d x) \\
& =\iint \overline{G_{\kappa}(x-y)} u(y) \overline{v(x)} V(d y) V(d x) \\
& =\iint u(y) \overline{G_{\kappa}(x-y) v(x)} V(d y) V(d x)=(u, Q(z) v),
\end{aligned}
$$

where we are using the fact that the Green's function for $R(\bar{z})$ is the complex conjugate of that for $R(z)$. This combined with the fact that $Q(z)$ is bounded for $z \in \rho(H)$ shows that (d) holds.

We now turn our attention to verifying condition (e) of Theorem 2.1. Condition (e)(2) follows from Theorem 2.2, thus it only remains to verify the uniform continuity of $Q(z)$ for some appropriately chosen $\Lambda \subset \mathbf{R}$. To achieve this we shall let $V^{N}$ (respectively $V_{N}$ ) be the measure generated by restricting $V$ to outside (respectively inside) the ball $B_{N}$ of radius $N$ in $\mathbf{R}^{n}$. We let $A^{N}$ (respectively $A_{N}$ ) be the associated identification operators.

We then set $Q_{N}(z)=A_{N}\left[A_{N} R(\bar{z})\right]^{*}$ and let $\Lambda=\mathbf{R}-\{0\}$. We next provide

Lemma 4.5. Let conditions (1.3) and (1.5) hold for $V$ and suppose the support of $V$ is contained in the ball $B_{R}$ for some $R$. Let $c_{0}>0$ and set $\omega_{I}=\{s+i a$ : $\left.s \in I \subset \subset \Lambda, a<c_{0}\right\}$. Then $c_{I}\left(z_{1}, z_{2}\right) \leq N_{I}\left|z_{1}-z_{2}\right|$, where $N_{I}$ is a constant depending only on $I$ and

$$
c_{I}\left(z_{1}, z_{2}\right)=\sup _{y} \int_{\mathbf{R}^{n}}\left|G_{\kappa_{1}}(x-y)-G_{\kappa_{2}}(x-y)\right||V|(d x) .
$$

Proof. We use the estimate that follows easily from Thoe [21] that, for $z_{i} \in \omega_{I}$, $i=1,2$, there is a constant, $N_{R}$, depending on $R$ such that

$$
\left|G_{\kappa_{1}}(x)-G_{\kappa_{2}}(x)\right| \leq N_{R}\left|z_{1}-z_{1}\right||x|^{2-n}, \quad x \in B_{R} .
$$


For $x, y \in B_{R},|x-y| \leq 2 R$. Thus,

$$
\begin{aligned}
c_{I}\left(z_{1}, z_{2}\right) & =\sup _{y \in B_{R}} \int_{\mathbf{R}^{n}}\left|G_{\kappa_{1}}(x-y)-G_{\kappa_{2}}(x-y)\right||V|(d x) \\
& \leq \sup _{y \in B_{R}} \int_{\mathbf{R}^{n}} N_{2 R}\left|z_{1}-z_{2}\right||x-y|^{2-n}|V|(d x) \\
& =N_{2 R}\left|z_{1}-z_{2}\right| C M R^{n} \quad(\text { by }(3.17)) \\
& =N_{I}\left|z_{1}-z_{2}\right|,
\end{aligned}
$$

where $N_{I}=N_{2 R} C M R^{n}$.

With the aid of the above lemma we can now establish the uniform continuity of $Q_{N}(z)$ in $\omega_{I}$ with the following:

Lemma 4.6. Let $V$ be a measure on $\mathbf{R}^{n}$ satisfying the conditions of Lemma 4.5. Let $\omega_{I}$ be as above. Then $Q(z)=A(A R(\bar{z}))^{*}$ is uniformly continuous in $\omega_{I}$.

Proof. For $z_{1}, z_{2} \in \omega_{I}$,

$$
\begin{aligned}
\left|\left(\left[Q\left(z_{1}\right)-Q\left(z_{2}\right)\right] u, v\right)\right|^{2} \\
=\left|\iint\left(G_{\kappa_{1}}(x-y)-G_{\kappa_{2}}(x-y)\right) u(y) \overline{v(x)} V(d y) V(d x)\right|^{2} \\
\leq \iint\left|G_{\kappa_{1}}(x-y)-G_{\kappa_{2}}(x-y)\right||u(y)|^{2}|V|(d y)|V|(d x) \\
\quad \times \iint\left|G_{\kappa_{1}}(x-y)-G_{\kappa_{2}}(x-y)\right||v(x)|^{2}|V|(d y)|V|(d x) \\
=c_{I}\left(z_{1}, z_{2}\right)^{2}\|u\|^{2}\|v\|^{2} .
\end{aligned}
$$

We now apply Lemma 4.5 to conclude

$$
\left\|Q\left(z_{1}\right)-Q\left(z_{2}\right)\right\| \leq N_{I}\left|z_{1}-z_{2}\right| \text {. }
$$

This establishes the uniform continuity of $Q(z)$ on $\omega_{I}$.

Lemma 4.7. If the measure $V$ satisfies (1.4) and (1.5) or (1.6) and (1.5) and $Q(z)$ and $Q_{N}(z)$ are as above, then $Q_{N}(z)$ converges to $Q(z)$ in norm as $N \rightarrow \infty$. In addition, the convergence is uniform for $z \in \omega_{I}$.

Proof. We write

$$
\left.\mid\left(\left[Q(z)-Q_{N}(z)\right]\right) u, v\right)\left.\right|^{2}=\left|\left(A^{N}[A R(\bar{z})]^{*} u, v\right)+\left(A_{N}\left[A^{N} R(\bar{z})\right]^{*} u, v\right)\right|^{2}
$$

Examining the first term in (4.23) we have

$$
\begin{aligned}
\left|\left(A^{N}[A R(\bar{z})]^{*} u, v\right)\right|^{2} & \leq\left|\iint G_{\kappa}(x-y) u(y) \overline{v(x)} V(d y) V^{N}(d x)\right|^{2} \\
& \leq 4\left(I_{1}^{N}+I_{2}^{n}\right)
\end{aligned}
$$

where

$$
I_{1}^{N}=\left|\iint_{|x-y| \leq 1} G_{\kappa}(x-y) u(y) \overline{v(x)} V(d y) V^{N}(d x)\right|^{2}
$$


and

$$
I_{2}^{N}=\left|\iint_{|x-y|>1} G_{\kappa}(x-y) u(y) \overline{v(x)} V(d y) V^{N}(d x)\right|^{2} .
$$

Using our estimates for $G_{\kappa}$ for $z \in \omega_{I}$ we have

$$
\begin{aligned}
I_{1}^{N} \leq & \left\{\iint_{|x-y| \leq 1} c_{1}|(x-y)|^{2-n}|u(y)||v(x)||V|(d y)\left|V^{N}\right|(d x)\right\}^{2} \\
\leq & \iint_{|x-y| \leq 1} c_{1}|(x-y)|^{2-n}|u(y)|^{2}|V|(d y)\left|V^{N}\right|(d x) \\
& \times \iint_{|x-y| \leq 1} c_{1}|(x-y)|^{2-n}|v(x)|^{2}|V|(d y)|V|(d x) \\
\leq & c_{1}^{2} c\|u\|^{2}\|v\|^{2} \sup _{x>N} \int_{|x-y| \leq 1}|(x-y)|^{2-n}|V|(d y),
\end{aligned}
$$

where $c$ is the uniform bound on $\int_{|x-y| \leq 1}|x-y|^{2-n}|V|(d x)$ by assumption (1.5). By (1.5) we see the last integral in (4.26) vanishes as $N \rightarrow \infty$. Thus, $I_{1}^{N} \rightarrow 0$ uniformly in $\omega_{I}$ as $N \rightarrow \infty$.

Turning our attention now to $I_{2}^{N}$ and again using the Green's functions estimates we have

$$
\begin{aligned}
I_{2}^{N} & \leq\left\{\iint_{|x-y|>1} C_{1}|(x-y)|^{(1-n) / 2} e^{-\mathrm{im} \kappa|x-y|}|u(y)||v(x)||V|(d y)\left|V^{N}\right|(d x)\right\}^{2} \\
& \leq C_{1}^{2}\|u\|^{2}\|v\|^{2} \iint|x-y|^{1-n}|V|(d y)\left|V^{N}\right|(d x),
\end{aligned}
$$

where $C_{1}=\sup _{z \in \omega_{I}}|\kappa|^{(3-n) / 2} c_{1}$. By the integrability condition (1.6) we see the last integral above vanishes as $N \rightarrow \infty$. Thus $I_{2}^{N} \rightarrow 0$ uniformly in $\omega_{I}$ as $N \rightarrow \infty$.

If $V$ satisfies (1.4), then $I_{2}^{N}$ satisfies

$$
\begin{aligned}
I^{N} 2 & \leq\left\{\iint_{|x-y|>1} C_{1}|(x-y)|^{(1-n) / 2} e^{-\operatorname{im} \kappa|x-y|}|u(y)||v(x)||V|(d y)\left|V^{N}\right|(d x)\right\}^{2} \\
& \leq C_{1}^{2}\|u\|^{2}\|v\|^{2} \sup _{y>N}\left\{\iint_{|x-y|>1}|(x-y)|^{(1-n) / 2}|V|(d x)\right\}^{2} .
\end{aligned}
$$

By condition (1.4) we see the last integral above vanishes as $N \rightarrow \infty$. Thus, $I_{2}^{N} \rightarrow 0$ uniformly in $\omega_{I}$ as $N \rightarrow \infty$ under either (1.4) or (1.6). Turning now to the second term in (4.23) we have

$$
\begin{aligned}
\left|\left(A_{N}\left[A^{N} R(z)\right]^{*} u, v\right)\right|^{2} & \leq\left|\iint G_{\kappa}(x-y) u(y) \overline{v(x)} V^{N}(d y) V_{N}(d x)\right|^{2} \\
& \leq 4\left(I_{3}^{N}+I_{4}^{N}\right)
\end{aligned}
$$

where

$$
I_{3}^{N}=\left|\iint_{|x-y| \leq 1} G_{\kappa}(x-y) u(y) \overline{v(x)} V^{N}(d y) V_{N}(d x)\right|^{2}
$$


and

$$
I_{4}^{N}=\left|\iint_{|x-y|>1} G_{\kappa}(x-y) u(y) \overline{v(x)} V^{N}(d y) V_{N}(d x)\right|^{2} .
$$

Similar estimating methods as those applied above reveal that $I_{3}^{N}$ and $I_{4}^{N}$ also vanish uniformly in $\omega_{I}$ as $N \rightarrow \infty$ under either (1.4) or (1.6).

Combining the above estimates we have in view of $(4.23)$ that

$$
\left\|Q(z)-Q_{N}(z)\right\|^{2} \leq 4\left(I_{1}^{N}+I_{2}^{N}+I_{3}^{N}+I_{4}^{N}\right)
$$

for all $z \in \omega_{I}$. Thus, $Q_{N}(z) \rightarrow Q(z)$ uniformly for $z \in \omega_{I}$.

Corollary 4.8. Under conditions (1.4) and (1.5) or (1.6) and (1.5), $Q(z)$ as above is uniformly continuous for $z \in \omega_{I}$.

Proof. The result follows since each $Q_{N}(z)$ is uniformly continuous in $\omega_{I}$, and $Q_{N}(z) \rightarrow Q(z)$ uniformly in $\omega_{I}$.

Completion of the proof of Theorem 1.1. From the above we have shown that all of the conditions (a)-(e) of Theorem 2.1 are satisfied. We now let $\Omega^{+}$and $\Omega^{-}$be any Hahn decomposition of $V$ into its positive and negative parts and define a multiplication operator, $E$, by the rule:

$$
E(x)= \begin{cases}1 & \text { if } x \in \Omega^{+} \\ -1 & \text { if } x \in \Omega^{-} \\ 0 & \text { otherwise }\end{cases}
$$

Then clearly $E$ is a bounded, selfadjoint operator on $\mathbf{K}$. Finally, it is easily verified that for $B=E A$,

$$
\int u(x) \overline{v(x)} V(d x)=(A u, B v)_{\mathbf{K}} .
$$

This completes our proof.

\section{REFERENCES}

1. S. Agmon, Spectral properties of Schrödinger operators and scattering theory, Ann. Scuola Norm Sup. Pisa 2 (1975), 151.

2. W. O. Amrein and V. Georgescu, Strong asymptotic completeness of wave operators for highly singular potentials, Helv. Phys. Acta 47 (1974), 517-533.

3. P. Constantin, Scattering for Schrödinger operators in a class of domains with noncompact boundaries, J. Funct. Anal. 44 (1981), 87-113.

4. E. B. Davies, Eigenfunction expansions for singular Schrödinger operators, Arch. Rational Mech. Anal. 63 (1976), 261-272.

5. A. Devinatz, Schrödinger operators with singular potentials, J. Operator Theory 4 (1980), 25-35.

6. R. Ford, The use of delta functions in scattering past an obstacle, Doctoral Dissertation, University of California, Irvine, 1989.

7. T. Ikebe, Eigenfunction expansions associated with the Schrödinger operator and their applications to scattering theory, Arch. Rational Mech. Anal. 5 (1960), 1-34.

8. _ On eigenfunction expansions connected with the exterior problem for the Schrödinger equation, Japan J. Math. 36 (1967), 33-35.

9. E. M. Il'in, The principle of limiting absorbtion and scattering on non-compact obstacles, Soviet Math. (Iz. Vuz) 28 (1984), 46-55. 
10. T. Kato, Schrödinger operators with singular potentials, Israel J. Math. 13 (1972), 135-148.

11. J. Kupsch and W. Sandhas, Moeller operators for scattering on singular potentials, Comm. Math. Phys. 2 (1966), 147-154.

12. S. T. Kuroda, Scattering theory for differential operators. III: Exterior problems, Lecture Notes in Math., vol. 448, Springer-Verlag, 1975, p. 277.

13. P. Lax and R. Phillips, Scattering theory, Academic Press, 1967.

14. D. B. Pearson, Time dependent scattering theory for highly singular potentials, Helv. Phys. Acta

15. A. G. Ramm, Scattering by obstacles, Reidel, Dordrecht, 1986.

16. M. Schechter, Spectra of partial differential operators, North-Holland, Amsterdam, 1986.

17. __ Operator methods in quantum mechanics, North-Holland, Amsterdam, 1981.

18. __ Selfadjoint realizations in another Hilbert space, Amer. J. Math. 106 (1984), 43-65.

19. N. A. Shenk, Eigenfunction expansions and scattering theory for the wave equation in an exterior domain, Arch. Rational Mech. Anal. 21 (1966), 120-150.

20. Y. Shizuta, Eigenfunction expansion associated with the operator $-\Delta$ in the exterior domain, Proc. Japan Acad. 39 (1963), 656-660.

21. D. W. Thoe, Eigenfunction expansions associated with Schrödinger operators in $R_{n}, n \geq 4$, Arch. Rational Mech. Anal. 26 (1967), 335-356.

22. R. Weder and J. M. Combes, $A$ new criterion for existence and completeness of wave operators and applications to scattering by unbounded obstacles, Comm. Partial Differential Equations 6 (1981), 1179-1223.

23. C. Wilcox, Scattering theory for the d'Alembert equation in exterior domains, Lecture Notes in Math., vol. 442, Springer-Verlag, 1975.

Department of Mathematics, University of California, Irvine, California 92717

Current address: Department of Mathematics and Statistics, California State University, Chico, California 95929 